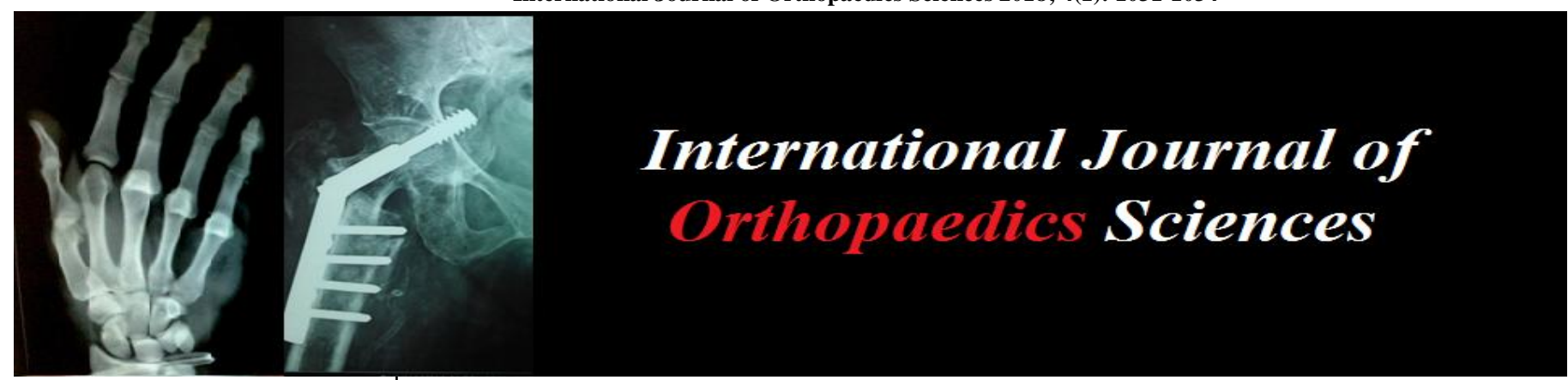

ISSN: $2395-1958$

IJOS 2018; 4(1): 1031-1034

(C) 2018 IJOS

www.orthopaper.com

Received: 22-11-2017

Accepted: 25-12-2017

Amol Babasaheb Parturkar MBBS DNB ORTHO, Registrar M.T. Agarwal hospital, Mumbai, Maharashtra, India

Pervez Shaikh MBBS DNB ORTHO, Registrar M.T. Agarwal hospital, Mumbai Mumbai, Maharashtra, India

Correspondence

Amol Babasaheb Parturkar MBBS DNB ORTHO, Registrar M.T. Agarwal hospital, Mumbai, Maharashtra, India

\section{Title of the manuscript: study of complications associated with plate fixation of middle one third clavicle shaft fracture}

\section{Amol Babasaheb Parturkar and Pervez Shaikh}

DOI: https://doi.org/10.22271/ortho.2018.v4.i1o.147

Abstract

1.1 Background: Both non-operative and surgical methods have been described for the management of this injury. Good outcome with high union rates and low complication rates has been reported with various surgical modalities. However, operative treatment has also got its own disadvantages.

1.2 Aim: To study the complications associated with plate fixation of middle one third clavicle shaft fracture.

1.3 Material and Methods: Thirty cases of mid-shaft clavicle fractures who underwent locking plate fixation were studied for complications and outcome. Those patients who attended regular follow-up and had recorded Constant-Murley Scores (CMS) were included.

1.4 Results: 28 fractures (93.3\%) united at or before three months. 6 patients had hypertrophic skin scar and in 5 patients plate prominence occurred out of which one underwent implant removal, delayed union in 2 patients and plate breakage in 1 patient and restricted range of movements in 1 patient.23 (76.6\%) had excellent functional outcome.

1.5 Conclusion: The low implant failure rate and complication rate in our study indicates that precontoured locking plate fixation is a safe procedure with excellent results in majority of patients.

Keywords: Mid-shaft clavicle fractures, Locking plate, complications, functional outcome

\section{Introduction}

Clavicle fracture is a common injury due to its subcutaneous and relatively anterior position. Fractures affecting the middle third account for majority of all clavicular fractures. They are classified into three groups based on their location along the bone. The middle-third fractures are most common and account for approximately $80-85 \%$ all clavicular fractures. ${ }^{[1]}$ The narrow cross section of the bone in the middle shaft combined with typical muscle forces acting over it predispose to fracture the bone in this locality. ${ }^{[2]}$ It is caused by either lowenergy or high energy impact.

Both non-operative and surgical methods have been described for the management of this injury. ${ }^{[3]}$ When displaced fractures are managed conservatively it may lead to suboptimal outcomes, functional impairment and a very high nonunion rates. ${ }^{[4,5]}$ Restoration of normal length and alignment by surgical methods can prevent these drawbacks of conservative treatment. Good outcome with high union rates and low complication rates has been reported with various surgical modalities of primary fixation of the displaced fractures. ${ }^{[6,7]}$ However, operative treatment has also got its own disadvantages such as surgical site infection, hypertrophic scar, hardware prominence and a repeat surgery for implant removal at times. The purpose of this study on clavicle fractures is to study the complications associated with plate fixation of middle one third clavicle shaft fracture.

\section{Material and Methods}

This prospective observational study included 30 patients who sustained clavicle mid-shaft fractures and were treated surgically with open reduction and internal fixation over a period of one year and followed up for next one year. 


\subsection{Inclusion criteria}

- $\quad$ Age group of 16-60 years.

- Middle third displaced clavicle fractures.

- All clavicle fractures threatening to pierce the skin/open fractures

\subsection{Exclusion criteria}

- Age less than 16 and more than 60

- Lateral end and medial third clavicle fractures

- Patients not fit or unwilling for surgery

After admission of the patient, detailed physical examination and required investigations were done. All patients were counseled about their injuries and treatment options. Decision regarding the operation was taken in consultation with patients after detailed counseling. Informed consent was obtained from all patients. Associated injuries, if any, were treated as per indicated treatment protocol.

All patients were operated under general anaesthesia. After minimal soft tissue and periosteum dissection, fracture fragments were reduced and fixed temporarily with Kirschner wire and then plate was applied over the superior aspect of the clavicle. (Lag screw was put wherever possible and then plate in neutralization mode was applied). While drilling from superior to inferior direction, a periosteal elevator was inserted inferiorly to prevent the drill from damaging the important neurovascular structures inferiorly. The medial and lateral fracture fragments were fixed with the plate with $3.5 \mathrm{~mm}$ cortical and locking screws depending upon the fracture morphology. At least three screws were put on either side of the fracture. Wound was closed in layers after ensuring proper hemostasis and sterile dressing was applied and broad arm sling was given.

After surgery limb was immobilized in a broad arm sling with shoulder held in adduction and internal rotation. Elbow was maintained at $90^{\circ}$ of flexion. Rehabilitation protocol was planned depending upon the stability of fixation. For wellfixed and stable fixation, active assisted range of motion of shoulder joint was allowed up to 90 degrees of abduction and flexion, 45 degrees of external rotation. After 6 weeks, patient was reassessed with X-ray and active range of motion in all planes was allowed. At eight to 12 weeks, isometric and isotonic exercises were prescribed to the shoulder girdle muscles. In comminuted fractures and fractures which were not rigidly fixed, range of movement exercises was delayed. Patients were followed up as outpatient basis with X-ray after 6 weeks, 3 months, 6 months and 1 year from the date of surgery. Clinical evaluation will be done by Constant and Murley scoring system. ${ }^{[6]}$

\section{Results}

Out of 30 clavicle mid-shaft fracture patients, 25 patients $(83.3 \%)$ had direct injury to shoulder and 5 patients (16.6\%)had indirect injury to shoulder. 21 patients $(70 \%)$ had road traffic accident, 4 patients $(13.3 \%)$ had simple fall on shoulder, 5 patients $(16.6 \%)$ had fall on outstretched hand.

Majority of the patients studied were of the age group 16-29 (14) that is $46.6 \%$. 27 patients $(90 \%)$ were males and 3 patients $(10 \%)$ were females.

Table 1: Patient characteristics

\begin{tabular}{|c|c|c|}
\hline Characteristics & No. of patients & Percentage \\
\hline Age group (years) & & \\
\hline $16-29$ & 14 & 46.6 \\
\hline $30-39$ & 2 & 6.6 \\
\hline $40-49$ & 5 & 16.6 \\
\hline $50-60$ & 9 & 30 \\
\hline Sex & & \\
\hline Male & 27 & 90 \\
\hline Female & 3 & 10 \\
\hline Side affected & & \\
\hline Right & 22 & 73.3 \\
\hline Left & 8 & 26.6 \\
\hline Associated injuries & & \\
\hline Head injury & 1 & 3.3 \\
\hline Chest injury & 3 & 10 \\
\hline Ankle fracture & 1 & 3.3 \\
\hline Scapula fracture & 3 & 10 \\
\hline Tendon injuries & 1 & 3.3 \\
\hline Forearm fracture & 1 & 3.3 \\
\hline
\end{tabular}

There were 17 patients $(56.6 \%)$ who were classified as type 2B $1,12(40 \%)$ as type $2 \mathrm{~B} 2$ and one (3.3\%) as type $2 \mathrm{~A} 2$. The most commonly used implant was anatomically contoured LCP which was used in 15 patients (50\%), followed by simple straight $3.5 \mathrm{LCP}$ which was used in 11 patients $(36.6 \%)$ (Table 2$)$.

Table 2: Classification of fractures

\begin{tabular}{|c|c|c|c|}
\hline \multicolumn{2}{|c|}{ Type } & No. of cases & Percentage \\
\hline \multirow{3}{*}{ Type -2 Middle Third } & A2 & 1 & 3.3 \\
\cline { 2 - 4 } & B1 & 17 & 56.6 \\
\cline { 2 - 4 } & B2 & 12 & 40 \\
\hline
\end{tabular}

For type II A 2, one simple LCP was used. For type II B 1, 1 DCP, 8 anatomically contoured LCP and 8 simple LCP were used. For type II B 2, 1 reconstruction plate, 2 DCP, 7 anatomically contoured LCP and 2 simple LCP were used. 7- holed plate was the most commonly used plate (16 patients$53.3 \%$ ) followed by 6 holed plate (12 patients-40\%) followed by 8 holed plate ( 2 patients- $6.6 \%$ ). 
Table 3: Fracture type and type of plate used

\begin{tabular}{|c|c|c|c|c|c|}
\hline \multicolumn{2}{|c|}{ Type of fracture (Robinson type) } & Reconstruction plate & DCP & Anatomically contoured LCP & Simple LCP \\
\hline \multirow{3}{*}{ Type II } & A2 & - & - & - & 1 \\
\cline { 2 - 6 } & B1 & - & 1 & 8 & 8 \\
\cline { 2 - 6 } & B2 & 1 & 2 & 7 & 2 \\
\hline
\end{tabular}

In this study, 27 patients $(90 \%)$ were treated within three days of the injury while 3 patients (10\%) were treated between 4-7 days. Average time duration between date of injury and date of surgery was 2 days with standard deviation of 1.6 days.

In middle third clavicle fracture 28 fractures $(93.3 \%)$ united at or before three months. 2 patients $(6.6 \%)$ had delayed union for which they underwent bone grafting. (one delayed union resulted in plate breakage).

Table 4: Complications

\begin{tabular}{|c|c|c|c|}
\hline Complications & Types & No. of cases & \% \\
\hline \multirow{4}{*}{ Minor } & Hypertrophic skin scar & 6 & 20 \\
\cline { 2 - 4 } & Plate prominence & 5 & 16.6 \\
\cline { 2 - 4 } & Delayed union & 2 & 6.6 \\
\cline { 2 - 4 } & Restricted ROM & 1 & 3.3 \\
\hline Major & Plate breakage & 1 & 3.3 \\
\hline
\end{tabular}

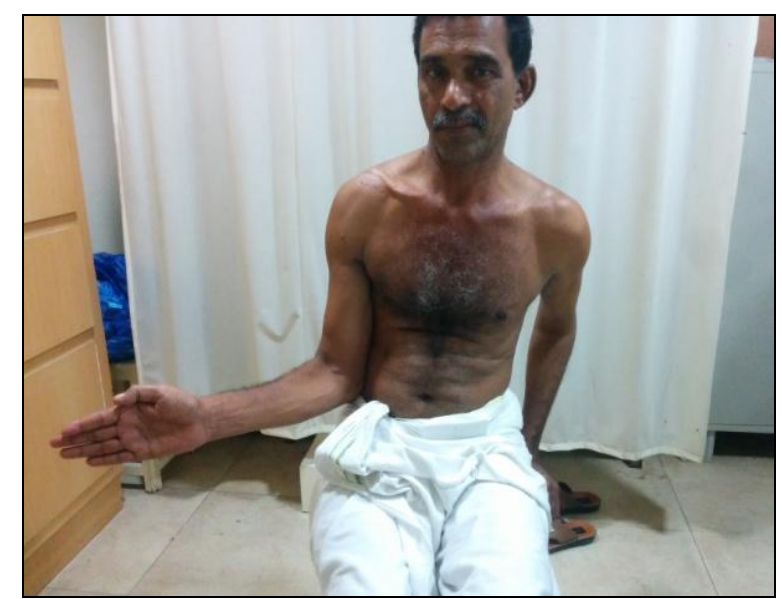

Fig 1: Plate prominence in postoperative patient of clavicle fracture.

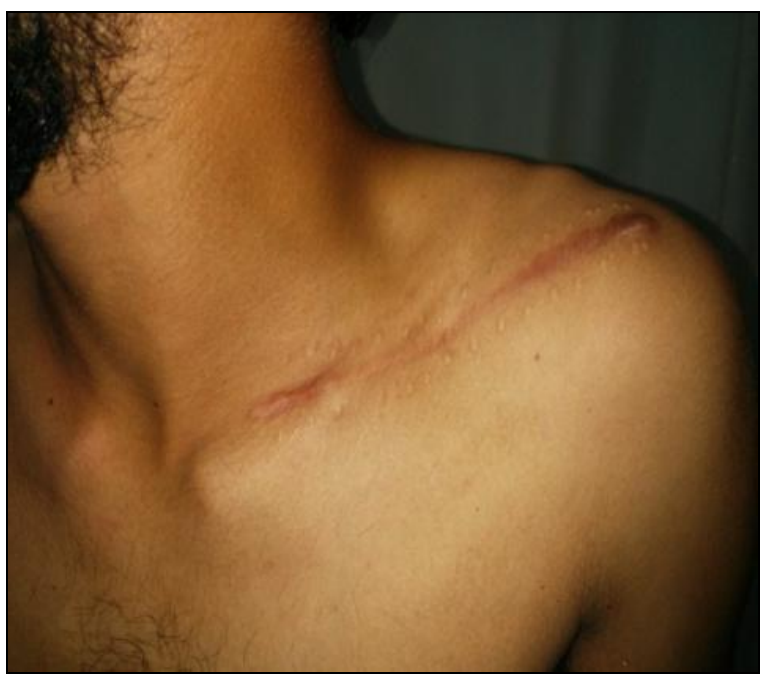

Fig 2: skin scar in postoperative patient of clavicle fracture.

In middle third clavicle fixation 6 patients had hypertrophic skin scar and in 5 patients plate prominence occurred out of which one underwent implant removal. In 2 patients delayed union occurred. In 1 patient delayed union lead to plate breakage. 1 patient $(3.3 \%)$ restriction of shoulder movements occurred. This was because of the reason that patient had sustained head injury and so was not compliant with physiotherapy protocol. Movements were improved once patient was recovered from head injury and underwent proper physiotherapy.

Functional outcome was assessed by Constant and Murley Score System (Table 3).

Table 5: Functional outcome

\begin{tabular}{|c|c|c|}
\hline Functional outcome & No. of fractures & $\mathbf{\%}$ \\
\hline Excellent & 23 & 76.6 \\
\hline Good & 5 & 16.6 \\
\hline Fair & 2 & 6.6 \\
\hline Poor & 0 & 0 \\
\hline Total & 30 & 100 \\
\hline
\end{tabular}

\section{Discussion}

The present study was done with an aim to look at the complications, re-operation rates and functional outcome in patients surgically treated for middle third shaft clavicle fractures.

There were 17 patients $(56.6 \%)$ who were classified as type 2B1, 12 (40\%)as type 2B 2 and one (3.3\%) as type 2A2.Bostman et al. also reported Robinson type-2B1 as common type in 81 patients $(78.64 \%) .{ }^{[8]}$

In our study, 28 fractures $(93.3 \%)$ united at or before three months. 2 patients $(6.6 \%)$ with delayed union underwent bone grafting. 1 patient with delayed union had plate breakage. Shen $\mathrm{W} \mathbf{J}$ et al. in their stated radiological union occurred approximately between 6 to 12 weeks. ${ }^{9}$ In a study by Lazarus $\mathrm{MD},{ }^{[10]}$ union of the fracture occurred between 6-12 weeks. In a study by Cho et al., ${ }^{[11]}$ bony union time was 13.2 weeks and 14.6 weeks for locking compression plate fixation and reconstruction plate fixation respectively.

One patient $(3.3 \%)$ plated with reconstruction plate for Robinson type $2 \mathrm{~B} 2$ fracture had plate breakage in postoperative period. He was re-operated with anatomically contoured LCP with bone grafting. This fracture united at 20 weeks postoperatively. In Bostman et al. study 2 patients treated with semi tubular plate had implant breakage at $2^{\text {nd }}$ and $7^{\text {th }}$ postoperative weeks respectively. ${ }^{[8]}$ Both cases were treated by re-plating using dynamic compression plate with bone grafting.

Two patients $(6.6 \%)$ had delayed union. One case of delayed union resulted in plate breakage for which re-plating and bone grafting was done. It united at the end of 20 weeks. For second patient with delayed union bone grafting was done which united at 16 weeks. In Bostman et al. study delayed union occurred in 3 Patients $(2.91 \%)$. ${ }^{[8]}$ Erborgt et al. reported the surgical outcome after open reduction and plate fixation of middle-third fractures of the clavicle in 39 semiprofessional athletes. Wound infection was seen among $18 \%$ of the cases, $5 \%$ of the cases had nonunion, $7 \%$ of the cases had neurological symptoms and $5 \%$ of the cases had refracture. ${ }^{[12]}$

In this study, majority of the patients i.e., 23 (76.6\%) had excellent functional outcome. A study by Campochiaro et al. noted that excellent clinical outcomes can be achieved in cases with mid-shaft clavicle fractures treated with locking 
plate osteosynthesis. ${ }^{[13]}$ In a study by Agarwal et al., $8.35 \%$ cases had excellent outcome with 4 to 5 weeks time to return to functional range.

The low implant failure rate and complication rate in our study indicates that pre-contoured locking plate fixation is a safe procedure with excellent results in majority of patients.

\section{References}

1. Stanley D, Trowbridge EA, Norris SH. The mechanism of clavicular fracture. A clinical and biochemical analysis. J Bone Joint Surg Br. 1988; 70B:461-464.

2. Craig EV, Basamania CJ, Rockwood CA. Fractures of the clavicle. Chapter 11. In: Rockwood CA, Matsen FA, Wirth MA, Lippitt SB, editors, The shoulder. $3^{\text {rd }}$ edition Philadelphia: Saunders, 2004, 455-519.

3. Neer CS. Nonunion of the clavicle. JAMA 1960; 172:1006-1011.

4. Hill JM, McGuire MH, Crosby LA. Closed treatment of displaced middle-third fractures of the clavicle gives poor results. J Bone Joint Surg Br. 1997; 79B:537-539.

5. Zlowodzki M, Zelle BA, Cole PA, et al. Treatment of mid-shaft clavicle fractures: systemic review of 2144 fractures. J Orthop Trauma. 2005; 19:504-507.

6. Canadian Orthopaedic Trauma Society (MD McKee, principal investigator). Plate fixation versus non operative care for acute, displaced mid-shaft fractures of the clavicle. J Bone Joint Surg. 2007; 89A:1-11.

7. Chen $\mathrm{CH}, \mathrm{Ch} \mathrm{WJ}$, Shih $\mathrm{CH}$. Surgical treatment for distal clavicle fractures with coraco-clavicular ligament disruption. J Trauma. 2002; 52:7-8.

8. Bostman O, Manninen M, Pihlajamaki H. Complications of plate fixation in fresh displaced mid clavicle fractures. J Trauma. 1997; 43:778-783.

9. Shen WJ, Liu TJ, Shen YS. Plate fixation of fresh displaced mid shaft clavicle fractures Injury. 2000; 31(3):175-179.

10. Lazarus MD. Fractures of the clavicle. Chapter 26, In: Bucholz RW and Heckma JD, Editors, Rockwood and Green's fractures in adults, 5th Edition, Philadelphia: Lippincott Williams and Wilkins. 2001, 1041-1078.

11. Cho CH, Song KS, Min BW, Bae KC, Lee KJ. Operative treatment of clavicle mid-shaft fractures: Comparison between reconstruction plate and reconstruction locking compression plate. Clin Orthop Surg. 2010; 2(3):154159.

12. Erborgt $\mathrm{OV}$, et al. Plate fixation of middle-third fractures of the clavicle in the semi-professional athlete. Acta Orthop. Belg. 2005; 71:17-21.

13. Campochiario G, Tsasis C, Gazzotti G, Rebuzzi M, Catani F. Displaced mid-shaft clavicular fractures: surgical treatment with a pre-contoured angular stability plate. Musculoskeletal Surg. 2012; 96(Suppl 1):21-26.

14. Agarwal T, Bhugra H, Jadhav S, Saurav K, Bhugra A. Operative treatment of mid-shaft clavicle fracture by locking plate. Indian Journal of Orthopaedic Surgery. 2017; 3(4):356-359. 